Revista Alicantina de Estudios Ingleses, núm. 2 (1989), pp. 101-117

\title{
Same/mismo y otros identificadores
}

\author{
José Ramón Losada Durán \\ Universidad de Santiago
}

\begin{abstract}
This paper offers a contrastive analysis of the identifiers same and mismo. It begins with a discussion of the morphological and distributional features that characterize them and distinguish one from the other. The Spanish adjective has more flexibility of distribution than the English one. The notion of identification is carefully explained, defined and differentiated from that of equality or likeness. Same and mismo are the terms mostly used to express the idea of identification and, therefore, they are syntactically and semantically analysed in detail. They are compared with words of similar meaning such as like/como, equal/igual, identical/idéntico and distinguished from them. Finally, the nominalization of the two adjectives, same and mismo, is also discussed and contrasted. The greatest difference between the two substantivized identifiers are found not at the level of meaning but at the level of morphology.
\end{abstract}

\section{Morfologia y distribución}

En sus aspectos morfológicos, same y mismo presentan las diferencias que habitualmente se observan entre el adjetivo inglés y el español: el primero carece de morfemas concordantes, permanece invariable cualquiera que sea el género del nombre con el que se agrupa, y el segundo muestra morfemas de género y de número:

$\begin{array}{lll}\text { The same } & \begin{array}{l}\text { man } \\ \text { woman }\end{array} & \begin{array}{l}\text { el mismo hombre } \\ \text { la misma mujer }\end{array} \\ \text { men } & \text { los mismos hombres } \\ \text { women } & \text { las mismas mujeres }\end{array}$

Como la mayoría de los adjetivos españoles mismo (y sus variantes) admite morfemas derivados de diminutivo y la formación del superlativo. A su equivalente inglés no le es dado unirse a esta clase de sufijos, sino solamente le es permitido agruparse con intensificadores independientes:

Hoy dijo lo mismito que ayer

Today he said exactly the same as yesterday 
They said the very same thing about my father

Dijeron la mismisima $\operatorname{cosa}(=$ lo mismisimo $)$ de mi padre

Los dos se encuentran integrados en la estructura de la frase nominal donde actúan como identificadores o intensificadores. Su distribución es muy desigual. Same va siempre en posición prenuclear, cualquiera que sea su misión en el sintagma. Mismo ocupa posición prenuclear cuando cumple la tarea de identificador. Con valor intensivo y ligado a las formas tónicas de los pronombres personales, a las del demostrativo, a las del posesivo y a algunos adverbios va siempre pospuesto. Cuando intensifica a un sustantivo, su posición puede ser prenuclear o posnuclear. Los dos se sustantivan por medio del artículo definido en cuyo caso se constituyen en unidades autónomas y actúan como núcleos. Veamos algunos ejemplos:

.. la masa es lo que no actúa por si misma ${ }^{1}$

... the mass is that part which does not act of itself $^{2}$

... el individuo trata de interceptar su propia visión de lo real, de su misma vida ${ }^{3}$

... through these notions the individual is trying to cut off any personal vision of reality, of his own very life

No one save Axel himself could find anything ${ }^{5}$

Nadie salvo el mismo Axel podía encontrar nada

I will see the town by myself ${ }^{6}$

Veré la ciudad por mí misma

Es obvio que el adjetivo español con valor intensivo abarca mayor número de elementos que su equivalente inglés, same, el cual sólo se realiza como intensificador agrupado con las formas del demostrativo. En este campo apenas existe correspondencia entre ellos; el inglés debe acudir a otros intensificadores para cubrir el territorio hacia el cual mismo ha adelantado su dominio.

En este trabajo, dada la complejidad del tema, nos limitaremos a estudiar same y mismo con valor de identificadores, dejando los demás aspectos para otro lugar.

\section{La identificación y sus instrumentos verbales}

El término identification/identificación tiene más de una acepción en cada una de las dos lenguas. E. Coseriu engloba la identificación en el proceso de la determinación nominal. Esta comprende cuatro tipos de operaciones: actualización, discriminación, delimitación e identificación. La operación determinativa fundamental -e idealmente primaria- es, sin duda, la actualización. Los nombres que integran un saber linguístico no son "actuales" sino "virtuales"; no significan objetos sino conceptos."7 Para actualizar un nombre es necesario dirigir los signos hacia los objetos. La identificación, en cambio, no orienta un signo conceptual hacia el ámbito de los objetos; no presenta los entes denotados como ejemplos o como representantes de un tipo o también como 
porciones de un objeto extenso, todo lo cual compete a la descriminación; tampoco modifica las posibilidades del signo limitando su denotación en sentido extensivo o intensivo como la limitación, sino que orienta al oyente hacia un valor semántico:

La identificación es la operación mediante la cual se especifica el significado de una forma <multívocas con la finalidad de asegurar su comprensión por parte del oyente actual o eventual. ${ }^{8}$

Los identificadores pueden ser ocasionales (<Córdoba >, Argentina), o usuales (<Castellón de la Plana >). Los dos últimos se distinguen de los otros tipos de determinadores en que son parte del signo. La identificación que describe Coseriu no se realiza con significados, sino con formas para asegurar la univocidad de un nombre que sin variar de forma designa más de un ser; convierte los nombres en unívocos haciendo que el oyente o lector les atribuya un sígnificado y no otros. Los identificadores más frecuentes están constituidos por frases nominales en aposición, frases preposicionales y adjetivos. No tienen otra misión que la de aportar una nota distintiva al nombre por la que se hace reconocible al oyente o lector.

No es éste el tipo de identificación en la que same y mismo realizan su tarea. Estrictamente, el concepto de identificación, igual que el de diferenciación, supone al menos dos términos. Al identificar, la mente examina y compara dos o más objetos, conceptos o seres animados para llegar a la conclusión de que son idénticos o de que son una y la misma cosa. El rasgo esencial que caracteriza a la identificación es que se parte de la multiplicidad y se termina en la unidad. En concreto, se trata de la unidad de referente. Dos realidades aprehendidas como distintas resultan ser una y la misma; dos nombres totalmente distintos denotan una sola persona:

Ribera y el Españoleto son la misma persona

Ribera and the Españoleto are the same person

La identificación presupone la búsqueda de la identidad de algo o de alguien. A su vez la identidad implica el conjunto de características que hacen a un ser reconocible y único. La identificación de una persona supone comprobar si es la misma que se dice o se busca. Se establece una dualidad: persona supuesta/persona que resulta ser, convergiendo al final en una y la misma.

Same y mismo son los instrumentos verbales -no los únicos- que con más frecuencia utilizan las dos lenguas respectivamente en el proceso que conduce a la identificación: "Mismo reproduce en el discurso una idea anterior, marca la identidad de la palabra con que se agrupa y su mención anterior. Necesita por ello de un término coincidente que aparece como antecedente o como complementación." $9 \mathrm{El}$ contenido de la cita anterior es aplicable en toda su extensión a same, según se deduce del paralelismo referencial que se detecta entre uno y otro. Los dos están dotados de análoga capacidad para identificar y llevan a cabo su tarea en condiciones análogas. Examinemos algunos ejemplos:

Las fronteras han servido para consolidar en cada momento la unificación política ya lograda. .. Pues bien: exactamente el mismo papel corresponde a la raza y a la lengua. ${ }^{10}$ 
Frontiers have served to consolidate at every stage the political unification already attained. . Exactly the same part is played by race and language.

En el enunciado anterior, same y mismo declaran que el papel que le corresponde a las fronteras y aquel que conjuntamente se le atribuye a la raza y a la lengua son idénticos. No hay más que una manifestación del sustantivo papel/part; la otra, la que actúa de antecedente, está implícita. Esta clase de elipsis es muy frecuente cuando el sustantivo al que acompañan same y mismo y el mencionado con anterioridad -el antecedente- son coincidentes. Conocido uno se infiere la existencia del otro, aunque no figure representado en el texto. En cambio, cuando los sustantivos son distintos es imprescindible que tengan mención expresa:

Josê Martínez Ruiz and Azorín are one and the same person

José Martínez Ruiz y Azorín son una y la misma persona

La unidad de referente en este caso está reforzada por one/una. Tienen únicamente valor enfático, no son necesarios para mantener el sentido de la construcción, puesto que same y mismo no dejan duda de que la persona designada por los dos nombres es idéntica. Esta construcción con one/uno (y sus variantes) es muy frecuente en estructuras coordinadas de una y otra lengua:

.. la rebelión de las masas es una y la misma cosa con el crecimiento fabuloso que la vida ha experimentado en nuestro tiempo. ${ }^{12}$

... the rebellion of the masses is one and the same thing as the fabulous increase that the human existence has experienced in our times.

Same y mismo van a menudo seguidos de cláusulas complementarias o frases preposicionales que contienen uno de los términos empleados en la identificacion de una realidad. Estas estructuras de complementación -a menudo implícitas- están introducidas por el pronombre relativo que y la preposición de en español y por un pronombre relativo that, which, who- o la conjunción as en inglés:

Pero este mito se volatiza enseguida sometiéndolo al mismo razonamiento que invalidó la comunidad de sangre y de idioma como fuentes de la nación. ${ }^{14}$

But this myth immediately disappears when submitted to the same reasoning which invalidates community of blood and language as originators of the nation.

Por encima de las más tiernas praderas, Eliacim, vuela del mismo color del aire, cse puro sentimiento que no consigo tener hacia tí. 16

Over softest meadows, Eliacim, flies that pure feeling the same colour as the air, which I can't manage to have toward you.

El hablante en primera persona utiliza a veces same y mismo con la cláusula de relativo para identificarse en dos actuaciones distintas:

.. I I, Amasa, the same that, used to paddle along the waterside to the schoolhouse. ${ }^{18}$ 
... Yo, Amasa, el mismo que remaba a lo largo de la orilla hacia el edificio de la escuela.

... I was the same person who took up the land for planting the said plantation at first. ${ }^{19}$

En las cláusulas de relativo el pronombre sustituye y evita la segunda aparición del sustantivo al que acompañan same y mismo, mientras que en las estructuras introducidas por as y de se encuentra implícito.

\section{La identificación y la igualdad}

La noción de identidad se encuentra próxima a la de igualdad. La una y la otra implican, al menos, la presencia de dos elementos y la ausencia de diferencia entre ellos. La distinción que las separa radica esencialmente en que la identificación requiere la unidad de referente, mientra que en la formulación de la igualdad existe más de uno. Los términos que intervienen en la expresión de la igualdad mantienen entre sí relación de reciprocidad que no suele detectarse entre los que participan en el proceso de identificación, a no ser que estén coordinados. Esta distinción ofrece criterios objetivos para discernir si se trata de una o de otra. Sin embargo, la frontera que las separa a veces se desvanece. En el ejemplo siguiente, las dos menciones del mismo sustantivo convergen en un solo objeto:

Your coat is the same colour as mine

Tu chaqueta es del mismo color que la mía

No hay duda de que el color de los dos objetos es uno solo y es idéntico. En otras construcciones, same y mismo más que identificar parecen expresar igualdad, semejanza o parecido. Alternan con like y como respectivamente sin que se observen cambios de significado:

(a) He walks the same as his father

$\mathrm{El}$ anda lo mismo que su padre

(b) He walks like his father

El anda como su padre

En (a) y en (b), same y mismo no tienen función adjetiva puesto que no preceden ni sustituyen a ningún sustantivo, antes bien modifican la acción de un verbo. Actúan como adverbios e indican que la manera de andar que se observa en el hijo es, más o menos, igual que la de su padre.

Por su contenido léxico equal e igual expresan la igualdad entre dos o más términos. El concepto de igualdad admite una escala gradual de mayor o menor coincidencia en los objetos que se comparan. Cuando los términos relacionados son coincidentes entre sí, equal e igual se aproximan de tal modo a same y mismo que éstos alternan con los anteriores:

(c) The two bottles are exactly equal in size 
Las dos botellas son exactamente iguales de tamaño

(d) The two bottles are exactly the same in size

Las dos botellas son exactamente del mismo tamaño

Los indicadores de la igualdad equal e igual- y los identificadores -same y mismo- son sinónimos en (c) y (d), es decir, proclaman que el tamaño de las botellas es idéntico en las dos construcciones al tiempo que excluyen toda posible diferencia entre ellos. La coincidencia total de equal e igual con los identificadores se debe a la presencia de los intensificadores exactly/exactamente. Sin ellos equal e igual, señalarían un grado de igualdad menos preciso: el tamaño de las botellas es más o menos idéntico; same y mismo, en cambio, seguirían testificando que el tamaño de los dos objetos es totalmente coincidente.

Cuando la igualdad de los términos es exacta y precisa equal e igual alternan con same y mismo sin la presencia de intensificadores:

(e) Robert and Michael are of equal height

Roberto y Miguel son iguales de altura

(f) Robert is the same height as Michael

Roberto tiene la misma altura que Miguel

La alternancia se fundamenta en que los términos relacionados son idénticos. No permiten establecer diferencias entre ellos: la altura de una persona es idéntica a la de la otra. Esto se pone aun más de manifiesto en matemáticas donde la igualdad de las fracciones o cantidades es tan exacta que necesariamente deben coincidir:

Four times five is equal to twenty

Cuatro veces cinco es igual a veinte

Two and two are equal to four

Dos y dos es igual a cuatro

Igual en español tiene mayor amplitud y flexibilidad de empleo que su análogo en inglés. Su alternancia con mismo es más frecuente que la que se observa entre equal y same:

Las caras de los orientales son todas igual(es) (=lo mismo)

The orientals' faces are alike

El empleo de same en correspondencia con igual(es) (=lo mismo: The orientals' faces are the same) es inapropiado, porque no deja margen para establecer diferencias que el hablante español presupone que existen en mayor o menor medida. Tal vez fuera más exacto decir: 
Las caras de los orientales parecen todas iguales

The orientals' faces seem to be the same

The same y lo mismo se realizan también como adverbios e introducen construcciones de estructura y sentido comparativo. Su tarea en estas estructuras no es la de identificar, sino la de expresar un concepto de igualdad o semejanza, más o menos preciso:

I am expected to treat Linda the same as I would any other student

Se espera que trate a Linda lo mismo que a cualquier otra estudiante

Independientemente de la función gramatical que puedan desempeñar, same y mismo pueden ser modificados por un grupo de adverbios -el último además por los sufijos del grado superlativo- que intensifican o atenúan su efecto identificador:

This is the very same song I heard yesterday

Esta es la mismísima canción que oí ayer

My camara is exactly the same size as yours.

Mi cámara tiene exactamente el mismo tamaño que la tuya

Silvia is almost the same height as Ruth

Silvia tiene casi la misma altura que Ruth

But the urgency felt, well-warrented or otherwise, was much the same 20

Pero la urgencia sentida, bien garantizada o lo contrario, era aproximadamente la misma

Igual ... que se utiliza como fórmula para expresar valores de igualdad o semejanza entre los términos que se contrastan. Concurre con lo mismo que y como y tiende a mostrarse invariable en estas circunstancias pero también aparece concordada.

En inglés corresponden a like, as, as well as:

Like a child who has no one to play with, she loitered through the dark hall ${ }^{2 I}$

Igual que (=lo mismo que, como) un niño que no tiene a nadie con quien jugar, ella vagaba por el oscuro salón.

... no se nutren sino de corazones frescos, de corazones sanos, de latidores corazones disfrazados, igual que alegres máscaras de bienaventuranza ${ }^{22}$

... [the birds] feed only on fresh hearts disguised, just like happy masks, by bliss ${ }^{23}$

Tú sabes bien, Eliacim, que los muebles convertibles, esos muebles que lo mismo sirven para un roto que para un descosido, son más prácticos que elegantes 
You know very well, Eliacim, that conyertible furniture, the furniture which does as well for one person as for other is more practical ${ }^{25}$

Instintivamente, lo mismo que el náufrago, buscará a que agarrarse. . 26

Instinctively, as do the shipwrecked he will look round for something to which to cling. . . 27

Equal e igual aparecen, además, con función sustantiva en singular y en plural, precedidos unas veces por el artículo definido o las formas del posesivo y otras veces solos:

He is such a good politician that he has no equal

Es tan buen político que no tiene igual

The Japanese workers in this factory have no equals

Los trabajadores japoneses de esta fábrica no tienen igual(es)

He likes to speak to his equals

Le gusta hablar con los iguales

She's your equal

Ella es tu igual

Algunos usos de igual no se corresponden con los de equal, por lo cual el último, en tales casos, es sustituido por el adjetivo even. La sustitución tiene lugar cuando (1) se hace referencia a la igualdad de un plano, superficie, nivel o altura:

I could come near them, the country being flat and even. . 28

Pude acercarme a ellos siendo el campo muy llano e igual. . .

The picture is even with the window

La foto está a igual altura que la ventana

El terreno era todo igual, sin altibajos

The ground was all even without any bumps

(2) Cuando expresa regularidad, paralelismo o igualdad como resultado final de un proceso:

His work is not very even

Su trabajo no es muy igual (= regular)

The two cars were even in the race

Los dos coches iban iguales en la carrera 
She does not like me and I do not like her, so we are even

Yo no le gusto a ella y ella no me gusta a mi así que estamos iguales serie:

(3) Cuando designa equilibrio de fuerzas o valores, y los números pares de una

The even pages of a book are on the left

Las páginas pares de un libro están a la izquierda

$2,4,6,8$ y 10 are even numbers

$2,4,6,8$ y 10 son números pares

The boxers were an even match

Los boxeadores eran parejos

En el apartado (3), no se da correspondencia entre igual e even pero el español utiliza palabras que implican la idea de igualdad.

Los adjetivos identical e idéntico (y sus variantes) se emplean a menudo como sinónimos de same y mismo para expresar la identidad. Como identificadores exigen la coincidencia exacta en todos los aspectos y detalles de los términos que relacionan. Implican la ausencia total de diferencia y, por ello, se muestran menos flexibles que same y mismo en cuanto a admitir intensificadores y diversidad de grados en la identificación de los objetos. Por esta razón no se suelen encontrar en las construcciones de estructura comparativa. Los modificadores que van situados a su derecha son generalmente frases preposicionales que contienen uno de los términos que intervienen en la identificación:

The hability to write is not identical with the hability to speak

La habilidad para escribir no es idéntica a la habilidad para hablar.

No two men have identical fingerprints

No hay dos hombres que tengan huellas dactilares idénticas

El español impone restricciones al empleo de este identificador como sustituto de mismo en posición prenominal, donde su equivalente inglés es gramaticalmente correcto y no disuena:

This is the identical place I stayed in last year

Éste es el *idéntico lugar donde yo estuve el año pasado

\section{La sustantivación de same y mismo}

Same y mismo no tienen función deíctica ni anafórica, es decir, por sí mismos carecen de capacidad para aludir a lo mencionado con anterioridad en el discurso y para 
señalar el distanciamiento de los objetos en el espacio y en el tiempo. Tampoco indican grados de proximidad o alejamiento respecto de las personas que interrvienen en el diálogo. Pero poseen la característica singular de agruparse con palabras que ejercen señalamiento deíctico o anafórico.

Siempre que same y mismo desempeñan función de identificadores van acompañados del artículo definido, ya que asociados con las formas del demostrativo tienen únicamente valor enfático. Ninguno de los dos se usa precedido del determinante cero. Los gramáticos ingleses consideran el empleo de same en tales casos como un vulgarismo limitado a la lengua coloquial y al estilo de la correspondencia comercial; en español, mismo no es gramatical:

There you have God the Father and God the Son. . . and God the Holy Ghost. Same substance. Same birth. ${ }^{30}$

Ahí tienes a Dios Padre y a Dios Hijo. . y a Dios Espíritu Santo. Misma sustancia. Mismo origen.

Your order is received with thanks; same shall have our best care.

Su pedido se recibe con agradecimiento; ${ }^{*}$ mismo será tratado con la máxima consideración.

Mismo puede aparecer acompañado del artículo indefinido pero siempre en libre alternancia con el indefinido.

Los dos perros son de una misma madre (= de la misma madre) pero no de un mismo padre (=del mismo padre)

The two dogs are from the same mother but not from the same father

La presencia del artículo definido es imprescindible dadas las características sintácticas y semánticas que distinguen a los dos adjetivos. ${ }^{31}$ Como a la identificación requiere al menos dos menciones de la misma entidaf. Por otra parte, los dos adjetivos, same y mismo, son inhábiles para ejercer ningún tipo de señalamiento hacia la primera aparición del sustantivo -el antecedente- $y$, en consecuencia, necesitan del concurso del artículo para que cumplan esa tarea.

Unidos al artículo definido en un mismo sintagma, el papel de los dos identificadores - same y mismo- consiste en establecer y destacar la unicidad de referente de las dos menciones de la misma palabra. La tarea del artículo, en cambio, es señalar anafóricamente la primera mención del sustantivo.

La frase nominal con estos componentes es siempre definida. Son bien conocidos los esfuerzos de los filósofos, siempre desde una perspectiva lógica, por aislar y fijar las características de las descripciones definidas. ${ }^{\mathbf{3 2}}$ Para Strawson, las descripciones definidas contienen dos presuposiciones fundamentales: existencia y unicidad. Así, la oración:

The regent of Spain is blond/El regente de España es rubio

presupone (1) que hay un regente en España; (2) que no hay más que un regente; (3) que es rubio. 
Searle analiza la "definidad" partiendo de la función de su referencia en el acto de la palabra. La esencia de la referencia definida se refleja en dos axiomas:

(a) Axioma de existencia: debe existir un solo y único objeto al que se le dirige la expresión del hablante, excluyendo cualquier otro.

(b) Axioma de identificación: La expresión del hablante debe contener la información suficiente para que a partir de ella el oyente identifique el objeto. ${ }^{34}$

Según lo expuesto, el concepto de definidad comprende esencialmente: (1) la "unicidad" de referente; (2) los datos para identificarlo. Estos dos supuestos se cumplen en el empleo y el significado de same y mismo, los cuales requieren, por una parte, la "unicidad" de referente, según expusimos en páginas anteriores, y, por otra, marcan la identidad de la palabra con la que se agrupan y su mención anterior. Por tener referencia definida, la frase nominal donde se encuentran same y mismo no puede tener sentido genérico; no comprende toda la clase, sino un miembro o un grupo específico de ellos.

El artículo con same y mismo actúa, además, como signo sustantivador que convierte a los dos adjetivos en unidades autónomas con valor funcional y denotativo de sustantivos. ${ }^{35}$

La sustantivación de estos dos adjetivos presenta diferencias importantes en las dos lenguas, que se localizan fundamentalmente en el plano morfológico. Los constituyentes del sintagma admiten morfemas de género y de número en español: $e l$ mismo, la misma; los mismos, las mismas, lo mismo. Por ellos conocemos si la frase nominal designa un ser masculino o femenino, o si se refiere a más de uno. Para el neutro, el adjetivo mismo conserva la terminación del masculino pero la forma lo del artículo aleja toda duda sobre su interpretación.

En cambio, los componentes del sintagma equivalente del inglés -the + samese encuentran morfológicamente indiferenciados; ninguno de los dos posee morfemas de género ni de número $\mathrm{y}$, por lo tanto, carecen de capacidad para indicar si el adjetivo sustantivado denota un ser o más de uno, si es del género masculino o femenino, o si, como el artículo neutro español, alude a un conjunto formado por aspectos de una o varias realidades. La frase nominal que resulta tras la asociación de los dos constituyentes es de interpretación ambigua, cuando el contexto no especifica la caracterización del referente:

$\ldots$ and the other two men said the same $e^{36}$

... y los otros dos hombres dijeron lo mismo

But the urgency felt, well-warranted or otherwise, was much the same 37

Pero la urgencia sentida, bien garantizada o lo contrario, era aproximadamente la misma

The marine the same who had been the sentinel without the cabin door. ..- entered it. .

El marino el mismo que había sido el centinela a la puerta del camarote. . .- entró en ella...

La terminación de los dos constituyentes -the + same- permanece invariable a pesar de que el sintagma denota tres realidades distintas en los tres últimos enunciados: en el primero, reproduce de manera abstracta un fragmento del discurso; 
en el segundo designa un concepto y en el tercero denota una persona concreta. Los sintagmas correspondientes del español, en cambio, contienen en sí todos los rasgos formales del nombre que denotan.

La sustantivación de same presenta características propias que no se encuentran en los demás adjetivos de la lengua. La sustantivación de los adjetivos ingleses es genérica o abstracta, nunca concreta e individualizante. Designa la clase o una realidad abstracta pero no puede referirse a un miembro aislado de la clase. Sintagmas como the rich/los ricos engloban a todas las personas que poseen la cualidad de ser ricos:

As is customary with the rich. . he apologized to the people, for his wealth, prosperity and elevated station. 38

Como es costumbre de los ricos. . él se disculpó ante la gente por su riqueza, prosperidad y posición elevada.

Cuando el hablante se dirige a un solo miembro de la clase, a un solo rico, el adjetivo pierde la sustantivación y retorna a su condición de adyacente prenominal: the rich man/el rico. En español, como acabamos de observar, la sustantivación puede ser abstracta, genérica o individualizante. A pesar de las diferencias morfológicas señaladas, same y mismo abarcan áreas denotativas análogas. Ninguno de los dos designa el género o la clase tomada en toda su extensión, sino uno o varios miembros de ella; denotan personas, objetos o conceptos introducidos con anterioridad en el texto o en la conversación. Los seres que son objeto de su referencia se encuentran siempre aislados de sus congéneres, claramente diferenciados e identificados. Su capacidad para aludir a lo mencionado en el discurso se extiende por igual a una unidad sintáctica, cualquiera que sea su nivel, que a varias tomadas en conjunto; a un elemento o a más de uno integrados en ellas. Reproducen y sustituyen:

(a) una frase nominal:

She looked the same under all circumstances

Parecía la misma en todas las circunstancias

Can I have a cup of coffee without milk, please

Give me the same, please

¿Me da una taza de café con leche, por favor?

-Deme a mí lo mismo, por favor

(b) un adjetivo:

Last winter was very cold and this is the same

El invierno pasado fue muy frío y este es lo mismo ${ }^{39}$

(c) Varias oraciones coordinadas sintetizadas de manera abstracta: 
Four men surrounded Robert and seized him by his arms and the collar of his coat, and then did the same with Emily

Cuatro hombre rodearon a Robert y lo cogieron por los brazos y por el cuello de la chaqueta y luego hicieron lo mismo con Emily.

The point is this: all of us who do not want war, do we want peace or do we want to be left in peace? Because it is not the same

$\mathrm{El}$ asunto es: todos nosotros que no queremos la guerra ¿queremos la paz o queremos que se nos deje en paz? Porque no es lo mismo

El empleo de same con valor de pronombre se encuentra reducido a la lengua comercial y a la de los documentos legales; ${ }^{\mathbf{4 0}}$ mismo con análoga función y sentido es más frecuente que same en inglés. La Real Academia llama la atención sobre el empleo abusivo que la prosa administrativa, periodística, publicitaria y técnica hacen de el mismo, la misma. Lejos de ser una fórmula elegante resulta mediocre y vulgar. ${ }^{41}$ Los dos -same y mismo- son anafóricos y sustituibles por un pronombre o las formás del posesivo

This charge was an error and we have struck same from the book

Este cargo fue un error y hemos borrado el mismo del libro

Thank you for the book; I shall return the same shortly

Gracias por el libro; devolveré el mismo en breve

Los ejemplos que ilustran las páginas anteriores revelan que same difiere de los otros adjetivos ingleses en que puede referirse a un miembro concreto, definido, pero nunca al género ni a la clase en su totalidad. Los demás adjetivos en idénticas circunstancias tienen siempre referencia genérica, abarcan la totalidad de los individuos y no apuntan hacia ninguno determinado. Mismo se distingue de los demás adjetivos españoles en que su referencia no comprende toda la clase, sino un miembro o un grupo variable de ellos. Pero los dos identificadores -same y mismo- tienen el poder de reproducir de manera abstracta un fragmento del discurso.

La sustantivación abstracta, no lexicalizada, que lleva por signo distintivo la forma del artículo neutro lo en español no tiene en inglés ningún señalizador que la diferencie de la individualizadora. A pesar de su indiferenciación morfológica, el contenido de same y lo mismo es siempre definido, conocido del lector o del oyente puesto que, cualquiera que sea la extensión del fragmento del discurso al que aluden e identifican, aquél debe haber sido expuesto con anterioridad en el texto o en la conversación. El papel de los dos identificadores es señalar que lo expuesto en el antecedente coincide con lo que contiene el enunciado donde se encuentran. En las secuencias,

Lo mismo acontece con el estado ${ }^{42}$

The same is happening with the state ${ }^{43}$ 
abstraen una situación descrita por el autor previamente y la identifican con aquella en que se encuentra el estado/state. Los dos adjetivos garantizan que los aspectos diversos de una o varias realidades presentadas en el contexto y aquellos que ambos denotan son idénticos, aunque expresados de modo diferente.

Lo mismo con el valor que venimos discutiendo concurre con lo propio, si bien el último es menos frecuente en español contemporáneo. La fórmula correspondiente en inglés es the same o the same thing:

Lo propio acaece en los demás órdenes, muy especialmente en el intelectual ${ }^{44}$

The same thing is happening in other orders, particularly in the intelectual ${ }^{45}$

Es necesario advertir que la sustantivación del adjetivo same tiene un índice de frecuencia inferior al de mismo. El inglés, en general, rehuye la nominalización de los adjetivos porque ninguno de los constituyentes que los forman posee morfemas concordantes. Aunque la referencia de the same está claramente determinada por el contexto, los usuarios de la lengua acuden con frecuencia a otras fórmulas: repiten el sustantivo, utilizan otro equivalente o colocan el pronombre one a la derecha del adjetivo:

Al llegar el mendigo hemos dado en el fondo ¿Ante quién puede ése manifestar su primacía? Ante el mismo de quien recibe la limosna

When we reach the beggar it looks as if we have come to the bottom. To whom can this man display primacy? For one, to the same person from whom he receives alms.

Si la sustantivación es no lexicalizada, abstracta, a menudo utiliza el sustantivo genérico thing para hacer más explícito el contenido de the same:

Por eso, si revisamos a su luz todo el pasado de la filosofía hasta Kant, nos parecerá que todos los filósofos han dicho lo mismo.

Hence, if by its light, we examine the whole past of philosophy up to the time of Kant, it will seem to us that, at bottom, all philosophers have said the the same thing. ${ }^{49}$ El resto de ella cgncluía en sayo de velarte, calzas de velludo para las fiestas con sus pantuflas
de lo mismo. .

The rest of it went on a fine cloth doublet, velvet breeches and sleepers for holidays. . 51

Asociados con las formas de los pronombres demostrativos -this, these, that, those/éste, ése, aquél, aquello (y sus variantes)-, same y mismo tienen solamente valor enfático. Las formas del demostrativo identifican las personas u objetos señalando su distanciamiento en el espacio, en el tiempo o en relación con la persona que habla. Su capacidad para situar los elementos les permite presentarlos por primera vez en el texto y en la conversación y señalar anafóricamente hacia lo mencionado. El señalamiento que ejerce el demostrataivo, tanto en la dimensión del espacio como del tiempo, es siempre concreto y preciso; separan, localizan y muestran los objetos, a veces en presencia de los locutores, de tal modo que el oyente los identifica sin error. De ahí que same y mismo se vean reducidos al papel de meros enfatizadores de la identificación realizada por las formas del demostrativo. 
No es frecuente encontrarlos agrupados en un mismo sintagama, si bien es preciso notar que el índice de frecuencia es más bajo en inglés que en español:

Tres hombres enmascarados robaron un banco en Madrid. Esos mismos hombres fueron detenidos por la policía cinco días más tarde.

Three masked men robbed a bank in Madrid, and those same men were detained by the police five days later.

Ever since childhood he had seen these towns from trains on this same line. ${ }^{52}$

Desde la niñez él había visto estas ciudades desde los trenes de esta misma línea.

I'll make Will start teaching me this very evening. 53

Haré que Will comience a enseñarme esta misma tarde.

That same car was stolen before

Ese mismo coche fue robado antes

That same fellow was an unknown fool

Aquel mismo tipo era un necio desconocido

Same y mismo, en los ejemplos anteriores, actúan únicamente como enfatizadores. Si se eliminaran de la construcción el hablante o lector reconocería por igual las personas y los objetos hacia los que apuntan las formas del demostrativo. Resaltan la identidad de los elementos en el campo mostrativo y, al parecer, suenan menos redundantes en español que en inglés.

Same con el sentido que venimos discutiendo concurre con el intensificador very:

Pero esta misma sensación íntima de dominio y valía le llevará a querer predominar fuera de su especialidad 54

But this very inner feeling of dominance and worth will induce him to wish to predominate outside his speciality

\section{Conclusión}

$\mathrm{El}$ análisis contrastivo de same y mismo ha revelado que en el nivel morfológico existen grandes diferencias entre uno y otro. El primero, igual que los demás adjetivos ingleses, carece de variantes para indicar el género y el número, mientras que el segundo está dotado de morfemas concordantes como los adjetivos de cuatro terminaciones.

Los dos, sin embargo, tienen asignado el papel de identificadores en una y otra lengua; señalan la identidad de la palabra con la que se agrupan y su mención anterior. La identificación se caracteriza por poseer al menos dos términos y por la unicidad de referente. Este último rasgo es el que la separa de la noción de igualdad. Sin embargo, los términos que se contrastan en la igualdad a veces son coincidentes en cuyo caso equal e igual adquieren valor de identificadores y concurren con same y 
mismo. Éstos se emplean, además, con función adverbial en oraciones de estructura comparativa. En estas circunstancias, se aproximan a menudo a la noción de igualdad o semejanza y alternan respectivamentecon like/como o igual que.

Ninguno de los dos identificadores tiene función deíctica ni anafórica; por sí mismos carecen de capacidad para aludir a lo mencionado con anterioridad en el discurso ni para señalar el distanciamiento de los objetos en el tiempo y en el espacio. Pero van siempre agrupados con el artículo definido y el demostrativo que cumplen esa tarea.

Funcionan unas veces como adjetivos integrados en el sintagma nominal y otras como unidades autónomas, sustantivados. A pesar de la falta de caracterización morfológica, la referencia de same, cuando va sustantivado, es clara y precisa porque está determinada por el contexto. Su equivalente español tiene los morfemas concordantes que distinguen a los adjetivos de cuatro terminaciones con lo cual el objeto y la extensión de su referencia se encuentaran inequívocamente definidos.

El empleo de same con valor de pronombre se encuentra reducido a la lengua comercial y legal. Su equivalente español con esa función y significado es más frecuente y recubre una zona más amplia en la lengua hablada y escrita, aunque su uso no está recomendado por la Real Academia. En estas condiciones los dos son sustituibles por un pronombre.

Desde un punto de vista semántico, ofrecen un paralelismo perfecto: ninguno de los dos tiene sentido genérico, es decir, no puede referirse a toda la clase o a todo el género, sino a un miembro concreto o a un grupo definido; reproducen una frase nominal, un adjetivo, una oración o un fragmento del discurso de extensión variada.

Agrupados con las formas del demostrativo, same y mismo tienen únicamente valor enfático, no se realizan como identificadores. No es frecuente su empleo en ninguna de las dos lenguas pero el índice de frecuencia es inferior en inglés que en castellano.

\section{Notas}

1. Cfr. J. Ortega y Gasset, La rebelión de las masas (Madrid: Revista de Occidente, 1964), p. 189.

2. Cfr. The Revolt of the Masses, traducción anónima de La rebelión de las masas (London: Unwin Books, 1963), p. 88.

3. Ibidem, p. 240.

4. Ibidem, p. 120 .

5. Cfr. Sinclair Lewis, Main Street, American Library (New York: Signet Classics, 1961), p. 1002.

6. Ibidem, p. 36.

7. Cfr. E. Coseriu, "Determinación y entorno," en Teoría del lenguaje y lingüística general (Madrid: Gredos, 1967), pp. 282-323, p. 293.

8. Ibidem, p. 306

9. Cfr. Juan Alcina Franch y José Manuel Blecua, Gramática española (Barcelona: Ariel, 1982), p. 676.

10. Cfr. J. Ortega y Gasset, op. cit., p. 254.

11. Cfr. The Revolt of the Masses, op. cit., p. 128.

12. Cfr. J. Ortega y Gasset, op. cit., p. 203.

13. Cfr. The Revolt of the Masses, op. cit., p. 96. 
14. Cfr. J. Ortega y Gasset, op. cit., p. 253.

15. Cfr. The Revolt of the Masses, op. cit., p. 125.

16. Cfr. Camilo José Cela, Mrs. Caldwell habla con su hijo (Barcclona: Ediciones Destino 1969), cap. 131, p. 132.

17. Cfr. J. S. Berstein, Mrs. Caldwell Speaks to Her Son (Ithaca: Corncll University press, 1969), cap. 131 , p. 108 .

18. Cfr. H. Melville, Billy Budd and Other Tales, New American Library (New York, 1961), p. 177.

19. Cfr. D. Defoe, Robinson Crusoe (London: Juvenile Productions, sin fecha), p. 262.

20. Cfr. H. Melville, op. cit., p. 71.

21. Cfr. Sinclair Lewis, op. cit., p. 152.

22. Cfr. Camilo José Cela, op. cit., cap. 132, p. 133.

23. Cfr J. S. Bernstein, trad., op. cit., cap. 132, p. 109.

24. Ibidem, cap. 96, p. 94.

25. Ibidem, cap. 96, p. 75.

26. Cfr. J. Ortega y Gasset, op. cit., p. 240.

27. Cfr. The Revolt of the Masses, p. 140.

28. Cfr. D. Defoc, op. cit., p. 105.

29. Cfr. H. Poutsma, A Grammar of Late Modern English, Part II (Groningen, 1916), p. 926.

30. Cfr. G. Green, Monsignor Quixote (Harmondsworth: Penguin, 1984), p. 52.

31. Mismo se agrupa, además, con las formas del posesivo, pero en ese caso no actúa como identificador, sino que tiene valor intensivo. Alterna con el adjetivo propio (y sus variantes) y corresponde al enfatizador inglés own: Hice mi misma indumentaria ( $=$ mi propia indumentaria $) / 1$ made my own costume.

32. Vid. B. Rusell, "On Denoting," Mind, 14 (1905), 479-493.

33. Cfr. P. F. Strawson, "On Referring," Mind, 59 (1950), 320-344.

34. Cfr. J. Searle, Speech Acts (London: Cambridge University Press, 1969); citado por Christopher G. Lyons, "The Meaning of the English Definite Article," en The Semantics of Deteminers, Johan Vander Auwera, ed. (Baltimore: University Park Press, 1980), p. 82.

35. Vid. O. Jespersen, A Modem English Grammar (London, 1964), II, 272-275.

36. Cfr. D. Defoe, op cit. p. 236.

37. Cfr. H. Melville, op. cit., p. 71.

38. Cfr. N. Hawthorne, The House of the Seven Gables (New York: Signet Classic, 1964), p. 118.

39. Vid. R. Quirk, S. Greenbaum, G. Leech y J. Svartvik, A Comprehensive Grammar of the English Language (London: Longman, 1985), p. 873.

40. Cfr. Poutsma, op. cit., pp. 928-929.

41. Esbozo de una Nueva Gramática de la Lengua Española (Madrid: Espasa Calpc: 1973), p. 212.

42. Cfr. J. Ortega y Gasset, op. cit., p. 191.

43. Cfr. The Revolt of the Masses, op. cit., p. 98.

44. Cfr. J. Ortega y Gasset, op. cit., p. 73.

45. Cfr. The Revolt of the Masses, op. cit., p. 14.

23.

46. Cfr. F. Diaz Plaja, El español y los siete pecados capitales (Madrid: Alianza Editorial, 1973), p.

47. Inderwick J. Palmer, The Spaniard and the Seven Deadly Sins (London: Pan Books, 1967), p. 14.

48. Cfr. J. Ortega y Gasset, op. cit., p. 211.

49. Cfr. The Revolt of the Masses, op. cit., p. 100.

50. Cfr. Miguel de Cervantes, Don Quijote de la Mancha, Colección Austral (Madrid: Espasa Calpe, 1985), p. 19.

51. Cfr. Miguel de Cervantes, Don Quixote, versión inglesa de J. M. Cohen (Harmondsworth: Penguin, 1987), p. 31.

52. Cr. Sinclair Lewis, op. cit., p. 27.

53. Cfr. J. Ortega y Gasset, op. cit., p. 185.

54. Cfr. The Revolt of the Masses, op. cit., p. 85.

55. Cfr. Sinclair Lewis, op. cit., p. 89. 